'Escuela de Trabajo Social, Universidad de Tarapacá, Arica-Chile. Investigadora visitante en el Instituto de Economía, Geografía y Demografía del Consejo Superior de Investigaciones Científicas (CSIC), Madrid, España. 2Escuela de Psicología y Filosofía, Universidad de Tarapacá, Arica-Chile. asistente Social. Magíster en Psicología Social. Doctora en Trabajo Social.

'Psicólogo. Doctor en Psicología. ‘Alumno/a de Psicología.

Este artículo es parte del proyecto de Iniciación FONDECYT "Envejecimiento

con éxito, apoyo social y etnicidad en personas mayores de la región de Arica y Parinacota" (№11140020), financiado por la Comisión Nacional de Investigación Científica (CONICYT) del Gobierno de Chile.

Recibido el 9 de marzo de 2016, aceptado el 31 de enero de 2017.

Correspondencia a: Lorena Gallardo Peralta Escuela de Trabajo Social de la Facultad de Ciencias Sociales y Jurídicas. Universidad de Tarapacá. Avenida 18 de Septiembre, 2222. Arica (Chile). Teléfonos: 582205659 o 582205048 lgallardo@uta.cl

\section{Validación del inventario de envejecimiento exitoso en personas mayores chilenas}

\author{
LORENA GALLARDO-PERALTA ${ }^{1, A}$, ALEJANDRO \\ CUADRA-PERALTA ${ }^{2, \mathrm{~B}}$, XIMENA CÁMARA-ROJO ${ }^{2, \mathrm{C}}$, \\ BETTY GASPAR-DELPINO ${ }^{2, C}$, RENATO SÁNCHEZ-LILLO $^{2, \mathrm{C}}$
}

\section{Validation of the successful aging inventory in chilean older people}

Background: There is a paucity of adequate and validated instruments for the measurement of successful aging for geriatric researchers in Chile. Aim: To validate the "Successful Aging Inventory" (SAI) in Chilean older people. Material and methods: SAI was answered by 777 older people aged $70 \pm 7$ years (63\% women) living in urban and rural areas, including the highlands of a Northern desert region of Chile. Results: A Cronbach alfa of 0.92 was obtained for the general dimension of successful aging. The figures for each dimension included in the theoretical model (functional performance, intrapsychicfactors, gero-transcendence; spirituality; purpose and life satisfaction), and ranged from 0.66 to 0.91. Confirmatory factorial analysis showed that the original inventory model, fits with the data collected from Chilean people. Conclusions: Successful Aging Inventory (SAI) is a reliable and adequate inventory, which can be used in Chilean older people.

(Rev Med Chile 2017; 145: 172-180)

Key words: Aging; Geriatrics; Surveys and Questionnaires.
$\mathrm{E}$ 1 proceso de envejecimiento característico de muchas sociedades contemporáneas ha obligado a numerosas disciplinas de las ciencias gerontológicas a propiciar alternativas teóricas/prácticas que permitan promover un envejecimiento funcional en las personas mayores. En este sentido, uno de los conceptos más relevantes en las últimas décadas es el de envejecimiento exitoso ${ }^{1-5}$. Se trata de un concepto multidimensional que contiene y trasciende la salud física y mental, incorporando distintos factores biológicos, psicológicos y sociales ${ }^{6-10}$.

Suele entenderse por envejecimiento exitoso aquel que se produce con una baja probabilidad de padecer enfermedades o invalidez, alta capacidad de funcionamiento físico y cognitivo, y un alto compromiso con la vida, tanto en términos de relaciones interpersonales como de participación en actividades productivas ${ }^{2}$. La importancia que ha cobrado este concepto se debe a que no considera exclusivamente el envejecimiento como una etapa de declive o de pérdida progresiva e irreversible de las capacidades, sino que enfatiza la posibilidad de envejecer con un óptimo funcionamiento físico, cognitivo y social. Las personas pueden por tanto participar en acciones de promoción, cambio o trasformación de sus estilos de vida, evitando enfermedades y por tanto mejorando sus posibilidades de envejecer mejor ${ }^{5,11}$.

Existen diversos modelos teóricos del envejecimiento exitoso y por ende diversas maneras de medirlo $^{4,12}$. En general, los modelos más utilizados proceden de marcos teóricos propios de las ciencias biomédicas y las psicosociales. Recientemente, sin 
embargo, se ha producido un notable desarrollo de modelos multidimensionales ${ }^{4}$. Este desarrollo tiene su reflejo en los instrumentos o escalas diseñadas para medir este constructo, de entre las cuales suelen utilizarse aquellas que incluyen una perspectiva subjetiva y objetiva de este concepto, así como una perspectiva holística o multidimensional ${ }^{8}$. Precisamente desde esta mirada integradora, el presente artículo ofrece la validación del Inventario de Envejecimiento Exitoso (Successful Aging Inventory, SAI $)^{13}$ en personas mayores chilenas. El modelo que subyace a este inventario identifica cinco dimensiones del proceso, definidas en la Tabla 1. Junto con las dimensiones clásicas en la valoración del envejecimiento exitoso (funcionamiento físico y cognitivo) este inventario añade la dimensión de gerotrascendencia y espiritualidad, que en la investigación reciente se han mostrado fundamentales ${ }^{8,13-15}$. Se trata de un instrumento ampliamente utilizado en los últimos años ${ }^{16-18}$.

El inventario SAI se basa en la perspectiva teórica de $\mathrm{Flood}^{3}$, autora que define el envejecimiento con éxito como la adaptación satisfactoria a los cambios físicos y funcionales del envejecimiento, incorporando de manera explícita la experiencia de una conexión espiritual y/o un sentido de propósito en la vida. Esta conceptualización (en la cual se basa el diseño del SAI) se fundamenta en una revisión de la literatura en las ciencias de la salud y ciencias sociales. En términos generales, envejecer con éxito supone un proceso de adaptación funcional y psicosocial de las personas mayores a los cambios propios de la vejez ${ }^{19}$, frente a lo cual el análisis se focaliza en los elementos internos y externos que permitan a la persona mayor adaptarse con éxito a esta etapa.
Ciertamente, en la vejez se experimentan diversos problemas, desde enfermedades hasta la pérdida de autonomía, pasando por el debilitamiento de redes sociales o la soledad. En este contexto, existen diferencias en la capacidad de las personas para adaptarse a este nuevo escenario utilizando para ello sus recursos psicosociales ${ }^{19,20}$. Así, la teoría de Flood ${ }^{13}$ propone cinco áreas asociadas al proceso de envejecer con "éxito". La hipótesis central establece la capacidad de adaptación de las personas mayores para envejecer con éxito, a través de estos cinco mecanismos. De esta manera, cabe la posibilidad de que los mecanismos puedan adaptarse de manera sincronizada o no; ello determinará que la persona pueda o no envejecer con éxito en los dominios físicos, cognitivos y sociales. En términos más específicos, los mecanismos de desempeño funcional implicarían que la persona mayor es consciente de sus condiciones fisiológicas y físicas durante el proceso de toma de decisiones en su vida. Los factores intrapsíquicos evalúan el afrontamiento positivo y la capacidad de ser flexible y creativo en la resolución de problemas. La espiritualidad es evaluada en términos de los comportamientos y valores/creencias de carácter religioso/espiritual, en la medida en que se trata de factores que potencialmente constituyen un recurso de afrontamiento. La gerotrascendencia se refiere al cambio en la visión del mundo y de la vida, de forma que la persona mayor desarrolla una capacidad de estar en calma, tranquilo y ser creativo para poder trascender. Por último, el propósito y la satisfacción vital describen una eventual valoración positiva global de los elementos del bienestar en las personas mayores.

En Chile, el proceso de envejecimiento es una

Tabla 1. Definición conceptual de las dimensiones del SAI

\begin{tabular}{|ll|}
\hline Mecanismos de desempeño funcional & $\begin{array}{l}\text { Se evalúan la capacidad de conciencia y elección de la persona mayor como una } \\
\text { respuesta adaptativa a las pérdidas fisiológicas y físicas producto del envejeci- } \\
\text { miento }\end{array}$ \\
\hline Factores intrapsíquicos & $\begin{array}{l}\text { Se mide la capacidad de la persona mayor para adaptarse al cambio y resolver los } \\
\text { problemas propios de la vejez }\end{array}$ \\
\hline Gerotrascendencia & $\begin{array}{l}\text { Se evalúa la metaperspectiva de la persona mayor, es decir, si ésta está más cen- } \\
\text { trada en una visión del mundo cósmica y existencial o trascendental }\end{array}$ \\
\hline Espiritualidad & Se analizan comportamientos religiosos que puede manifestar la persona mayor \\
\hline Propósito y satisfacción con la vida & $\begin{array}{l}\text { Se evalúan ámbitos del bienestar en las personas mayores, tales como tener un } \\
\text { propósito en la vida, preocupación por otras generaciones y satisfacción vital }\end{array}$ \\
\hline
\end{tabular}


realidad patente en las últimas décadas, lo que ha generado diversos cambios sociales y sanitarios que se ven reflejados en diversos instrumentos de medición en el ámbito de las ciencias gerontológicas $^{21-23}$. Sin embargo, no se disponen de instrumentos adecuados y validados para la medición y evaluación del envejecimiento exitoso en nuestro país. En esta línea, el objetivo de este estudio es ofrecer la validación del Inventario de Envejecimiento Exitoso (SAI) en población chilena.

\section{Material y Método}

\section{Participantes}

La muestra está conformada por 777 personas mayores chilenas que residen en la región de Arica y Parinacota. Se trata de una muestra con un margen de error (bajo el supuesto de muestreo aleatorio) del 3,46\% para un nivel de confianza del 95\%. Para asegurar la representatividad, se recurrió a un muestro estratificado por sexo, etnia y lugar de residencia (rural y urbano). Características fundamentales de la muestra: el $63 \%$ son mujeres. En términos de edad, el 55\% tiene entre 60 y 69 años, el 34\% entre 70 y 79 años y el 11\% más de 80 años (edad media de 70 años y desviación típica de 7,12). Respecto al estado civil, $48 \%$ tiene pareja, ya sea en matrimonio o en convivencia. En cuanto a la residencia, el $87 \%$ vive en zona urbana (Arica). En términos de alfabetización, el 91\% sabe leer y escribir. En términos económicos, 45\% sigue trabajando con remuneración económica y el 55\% aporta el mayor ingreso al hogar (jefe de hogar). Por último, el 30\% declara pertenecer a un grupo étnico originario, siendo el Aymara el más frecuente ( $88 \%$ de los casos) (Tabla 2 ).

\section{Instrumentos}

El Inventario de Envejecimiento Exitoso (SAI) posee 20 ítems y utiliza una escala tipo Likert: (0) totalmente en desacuerdo a (4) totalmente de acuerdo, para declaraciones como "He sido capaz de lidiar con los cambios que le han ocurrido a mi cuerpo a medida que he envejecido" "Soy bueno para pensar nuevas formas de resolver problemas". La puntuación resultante varía de 0 a 80, las puntuaciones mayores indican un envejecimiento con éxito. El SAI tiene cinco dimensiones (Tabla 1). La versión original propone que las dimensiones

Tabla 2. Principales características de la muestra

\begin{tabular}{|llc|}
\hline Variable & \multicolumn{1}{c|}{ Categorías } & Frecuencia n (\%) \\
Sexo & Mujer & $488(63 \%)$ \\
Edad (por grupos) & Hombre & $289(37 \%)$ \\
& $60-69$ años & $430(55 \%)$ \\
Estado conyugal & $70-79$ años & $260(34 \%)$ \\
& 80 y más años & $87(11 \%)$ \\
\hline Residencia & Casado/a o con pareja & $370(48 \%)$ \\
& Soltero/a & $228(29 \%)$ \\
& Viudo/a & $179(23 \%)$ \\
Alfabetización & Urbana: Arica & $677(87 \%)$ \\
& Rural: Altiplano & $58(8 \%)$ \\
Trabajo & Rural: Valles & $42(5 \%)$ \\
& Analfabeto & $70(9 \%)$ \\
Jefatura del hogar & Sabe leer y escribir & $707(91 \%)$ \\
\hline Etnicidad & Sigue trabajando & $350(45 \%)$ \\
& No trabaja & $427(55 \%)$ \\
\hline
\end{tabular}


Inventario de envejecimiento exitoso en personas mayores chilenas - L. Gallardo-Peralta et al

gerotrascendencia y propósito/satisfacción vital se unen para conformar una dimensión que puede denominarse gerovida.

\section{Procedimiento}

Se estableció contacto con la autora del inventario ${ }^{13}$, que facilitó la versión en español y autorizó explícitamente su uso. Dicha versión fue diseñada a partir de un proceso de traducción y traducción reversa y su validez de contenido ha sido comprobada parcialmente ${ }^{26}$. Los resultados obtenidos en la fase del estudio piloto de la presente investigación mostraron la necesidad de realizar diversos cambios de redacción en las preguntas. En la Tabla 3 se presentan los cambios de redacción efectuados a la versión facilitada por la autora.

Tabla 3. Preguntas de la versión original y la versión a validar del SAI

\section{Versión original SAI}

1. Yo puedo hacer todas las cosas que necesito hacer, para cuidar de mi casa y de mí

2. He sido capaz enfrentar los cambios que han ocurrido a mi cuerpo conforme he envejecido

3. Yo miro hacia el futuro

4. Me siento capaz de enfrentar mi propio envejecimiento

5. Me siento capaz de hacer frente a los acontecimientos de la vida

6. Puedo solucionar los problemas

7. Soy bueno encontrando nuevas formas de resolver problemas

8. Me gusta hacer cosas nuevas o creativas. Factores intrapsíquicos

9. Estoy en un estado de ánimo agradable, bueno

10. Pienso en mis seres queridos que han fallecido y me siento cerca de ellos

11. Dedico tiempo a la oración o hacer algún tipo de actividad religiosa

12. Como ya he envejecido, mi forma de pensar sobre el mundo ha cambiado

13. Prefiero tener unos pocos amigos cercanos que tener muchos casuales

14. A veces puede haber dos respuestas correctas a un problema o situación

15. Una relación con Dios o un poder superior es importante para mí

16. Yo siento interés y preocupación por la nueva generación

17. Mi vida tiene sentido

18. En general estoy satisfecho con mi vida ahora mismo

19. Siento que tengo un propósito en este mundo

20. Siendo la edad que tengo ahora es tan buena o mejor que yo pensé que sería.

\section{Versión de validación SAI}

1. Me manejo para hacer las cosas necesarias para cuidar mi casa y de mí mismo

2. He sido capaz de lidiar con los cambios que le han ocurrido a mi cuerpo a medida que he envejecido

3. Espero con ganas el futuro

4. Me siento capaz de afrontar mi propio envejecimiento

5. Me siento capaz de afrontar los acontecimientos de la vida

6. Soy capaz de encontrar solución a los problemas

7 Soy bueno para pensar nuevas formas de resolver problemas

8. Disfruto haciendo cosas nuevas y creativas

9. Tengo un carácter agradable y positivo

10. Pienso en mis seres queridos que han fallecido y me siento cercano a ellos

11. Paso tiempo en oración o haciendo algún tipo de actividad religiosa

12. A medida que envejezco, mi forma de ver el mundo va cambiado

13. Prefiero tener pocos amigos cercanos a muchos superficiales

14. Algunas veces puede haber dos respuestas correctas para un problema o solución

15. Para mí es importante tener una relación con Dios o algún poder superior

16. Estoy interesado/preocupado por las próximas generaciones

17. Mi vida tiene sentido

18. En general estoy satisfecho con mi vida en este momento

19. Creo que tengo un propósito en el mundo

20. A mis años, mi vida es mejor de lo que esperaba 
Respecto al proceso de aplicación, en el mes de junio de 2015 se realizó un estudio piloto con 33 adultos mayores que participaban en algunos programas del Servicio Nacional de Adultos Mayores. Cabe señalar, que el 10\% de la muestra del estudio piloto declaró pertenecer a una etnia originaria (Aymara). Como se mencionó, tras esta aplicación se realizaron algunos cambios en la redacción y adaptación lingüística de ciertas preguntas del SAI, manteniendo en todo caso las 20 preguntas originales. El factor etnicidad no influyó en los cambios de redacción efectuados. Esta circunstancia se debe a que en la actualidad las personas mayores aymaras utilizan el español como lengua principal.

Una vez modificado el cuestionario piloto se dio paso al trabajo de campo durante los meses de julio y agosto de 2015. La aplicación del cuestionario se realizó a través de entrevista personal con una duración de 30 a 40 minutos, los cuestionarios fueron aplicados por profesionales de las disciplinas de Trabajo Social y Psicología. Esta investigación es parte del proyecto FONDECYT de Iniciación 11140020, por tanto, b el Comité de Ética de la Universidad de Tarapacá supervisó y aprobó los aspectos éticos del estudio. De especial importancia es señalar que se obtuvo por escrito el consentimiento informado de todas las personas participantes.

\section{Análisis de datos}

A partir de los objetivos del estudio se realiza un análisis fiabilidad y un análisis factorial confirmatorio del inventario. Para evaluar la fiabilidad del SAI se analizó la consistencia interna a través del cálculo del alfa de Cronbach de los 20 ítems, sus 5 dimensiones y del inventario total. Posteriormente, se realizó un análisis factorial confirmatorio del modelo teórico. La bondad del ajuste del modelo fue calculada a través de diversos índices. Junto con el cálculo del estadístico $\chi^{2}$ (con su respectivo valor $\mathrm{p}$ ), se calcularon los siguientes índices: raíz del error cuadrático medio de aproximación (RMSEA), índice comparativo de Bentler-Bonett (CFI), índice de ajuste corregido (AGFI), índice de Tucker Lewis (TLI), índice de ajuste incremental (IFI). Para RMSEA, valores por debajo de 0,05 indicarían buen ajuste, y valores por debajo de 0,07 sugerirían un ajuste aceptable ${ }^{24}$. Para CFI, AGFI y TLI valores por encima de 0,95 sugieren un buen ajuste ${ }^{25}$. Los datos se analizaron con la versión 19 de los programas SPSS y Amos.

\section{Resultados}

La consistencia interna, para la muestra general, varía entre 0,91 y 0,92 (valor del alfa si se elimina el ítem). En tanto, las correlaciones ítems- test varían entre $0,32-0,77$, no se advierten correlaciones inversas. La correlación más débil $(<0,32)$ es el ítem 13 y las más fuertes $(>0,75)$ son los ítems 5, 6, 17 y 18 (Tabla 5). En la Tabla 4 se observa que el alfa de Cronbach es de 0,92 para el inventario general y el rango observado varía entre 0,66 y 0,91 para cada una de las dimensiones (Tabla 4 y 5 ).

El análisis factorial pretende contrastar el modelo original del SAI, contemplando por tanto cinco dimensiones, a saber, "mecanismos de desempeño funcional", "factores intrapsí-

Tabla 4. Descriptivos de las dimensiones y fiabilidad del SAI

\begin{tabular}{|c|c|c|c|c|c|c|c|c|}
\hline Dimensión & $\begin{array}{c}\mathbf{N}^{\circ} \\
\text { ítems }\end{array}$ & Media & Mediana & D. T. & Mínimo & Máximo & $\begin{array}{l}\text { Correlación } \\
\text { ítem-test }\end{array}$ & $\begin{array}{l}\text { Alfa de } \\
\text { Cronbach }\end{array}$ \\
\hline D. funcional (DF) & 2 & 7,04 & 8,00 & 1,82 & 0 & 8 & 0,74 & 0,848 \\
\hline F. intrapsíquicos (FI) & 7 & 23,19 & 23,20 & 5,88 & 0 & 28 & 0,74 & 0,915 \\
\hline Gerotrascendencia (G) & 6 & 19,76 & 21,00 & 4,03 & 0 & 24 & 0,41 & 0,675 \\
\hline Espiritualidad (E) & 2 & 5,60 & 6,00 & 2,34 & 0 & 8 & 0,50 & 0,666 \\
\hline $\begin{array}{l}\text { Propósito/satisfacción } \\
\text { vital (P/SV) }\end{array}$ & 3 & 10,43 & 11,00 & 2,55 & 0 & 12 & 0,80 & 0,895 \\
\hline Puntaje total SAI & 20 & 66,05 & 70,00 & 13,73 & 0 & 80 & & 0,926 \\
\hline
\end{tabular}


Tabla 5. Resultados del análisis de correlación ítem-test y consistencia interna por cada pregunta del SAI, para la muestra general, por sexo e indígenas

\begin{tabular}{|c|c|c|c|c|c|c|c|c|}
\hline \multirow[t]{3}{*}{ Preguntas SAI } & \multicolumn{2}{|c|}{ Muestra total } & \multicolumn{4}{|c|}{ Muestra según género } & \multicolumn{2}{|c|}{ Muestra indígena } \\
\hline & \multirow{2}{*}{$\begin{array}{l}\text { Corre- } \\
\text { lación } \\
\text { ítem-test }\end{array}$} & \multirow[t]{2}{*}{$\begin{array}{l}\text { Alfa al } \\
\text { eliminar }\end{array}$} & \multicolumn{2}{|c|}{$\begin{array}{l}\text { Correlación } \\
\text { ítem- test }\end{array}$} & \multicolumn{2}{|c|}{$\begin{array}{l}\text { Alfa al } \\
\text { eliminar }\end{array}$} & \multirow{2}{*}{$\begin{array}{l}\text { Corre- } \\
\text { lación } \\
\text { ítem-test }\end{array}$} & \multirow[t]{2}{*}{$\begin{array}{l}\text { Alfa al } \\
\text { eliminar }\end{array}$} \\
\hline & & & Mujer & Hombre & Mujer & Hombre & & \\
\hline $\begin{array}{l}\text { 1. Me manejo para hacer las cosas } \\
\text { necesarias para cuidar mi casa y } \\
\text { de mí mismo (DF) }\end{array}$ & 0,654 & 0,921 & 0,630 & 0,681 & 0,904 & 0,937 & 0,624 & 0,915 \\
\hline $\begin{array}{l}\text { 2. He sido capaz de lidiar con los } \\
\text { cambios que le han ocurrido a mi } \\
\text { cuerpo a medida que he enveje- } \\
\text { cido (DF) }\end{array}$ & 0,711 & 0,920 & 0,645 & 0,786 & 0,904 & 0,935 & 0,590 & 0,916 \\
\hline 3. Espero con ganas el futuro (FI) & 0,670 & 0,920 & 0,627 & 0,738 & 0,904 & 0,936 & 0,643 & 0,915 \\
\hline $\begin{array}{l}\text { 4. Me siento capaz de afrontar mi } \\
\text { propio envejecimiento ( } \mathrm{FI})\end{array}$ & 0,756 & 0,919 & 0,713 & 0,812 & 0,902 & 0,935 & 0,662 & 0,914 \\
\hline $\begin{array}{l}\text { 5. Me siento capaz de afrontar los } \\
\text { acontecimientos de la vida (FI) }\end{array}$ & 0,765 & 0,919 & 0,712 & 0,830 & 0,902 & 0,935 & 0.670 & 0,914 \\
\hline $\begin{array}{l}\text { 6. Soy capaz de encontrar solución } \\
\text { a los problemas (FI) }\end{array}$ & 0,767 & 0,919 & 0,710 & 0,823 & 0,903 & 0,935 & 0,722 & 0,913 \\
\hline $\begin{array}{l}\text { 7. Soy bueno para pensar nuevas } \\
\text { formas de resolver problemas (FI) }\end{array}$ & 0,755 & 0,919 & 0,709 & 0,809 & 0,902 & 0,935 & 0,701 & 0,914 \\
\hline $\begin{array}{l}\text { 8. Disfruto haciendo cosas nuevas y } \\
\text { creativas (FI) }\end{array}$ & 0,713 & 0,919 & 0,660 & 0,766 & 0,903 & 0,935 & 0,648 & 0,915 \\
\hline $\begin{array}{l}\text { 9. Tengo un carácter agradable y } \\
\text { positivo (FI) }\end{array}$ & 0,524 & 0,923 & 0,501 & 0,554 & 0,907 & 0,939 & 0,545 & 0,947 \\
\hline $\begin{array}{l}\text { 10. Pienso en mis seres queridos que } \\
\text { han fallecido y me siento cercano } \\
\text { a ellos }(G)\end{array}$ & 0,420 & 0,926 & 0,394 & 0,450 & 0,910 & 0,941 & 0,396 & 0,921 \\
\hline $\begin{array}{l}\text { 11. Paso tiempo en oración o ha- } \\
\text { ciendo algún tipo de actividad } \\
\text { religiosa }(\mathrm{E})\end{array}$ & 0,393 & 0,928 & 0,383 & 0,397 & 0,913 & 0,944 & 0,379 & 0,923 \\
\hline $\begin{array}{l}\text { 12. A medida que envejezco, mi forma } \\
\text { de ver el mundo va cambiado }(G)\end{array}$ & 0,439 & 0,926 & 0,424 & 0,458 & 0,910 & 0,941 & 0,477 & 0,919 \\
\hline $\begin{array}{l}\text { 13. Prefiero tener pocos amigos cer- } \\
\text { canos a muchos superficiales }(G)\end{array}$ & 0,321 & 0,927 & 0,304 & 0,357 & 0,912 & 0,942 & 0,356 & 0,921 \\
\hline $\begin{array}{l}\text { 14. Algunas veces puede haber } \\
\text { dos respuestas correctas para un } \\
\text { problema o solución }(G)\end{array}$ & 0,416 & 0,926 & 0,371 & 0,491 & 0,911 & 0,940 & 0,487 & 0,918 \\
\hline $\begin{array}{l}\text { 15. Para mí es importante tener una } \\
\text { relación con Dios o algún poder } \\
\text { superior }(E)\end{array}$ & 0,501 & 0,924 & 0,485 & 0,508 & 0,908 & 0,941 & 0,566 & 0,916 \\
\hline $\begin{array}{l}\text { 16. Estoy interesado/preocupado por } \\
\text { las próximas generaciones }(\mathrm{G})\end{array}$ & 0,527 & 0,923 & 0,488 & 0,569 & 0.907 & 0939 & 0,607 & 0,915 \\
\hline 17. Mi vida tiene sentido (P/SV) & 0,779 & 0,919 & 0,720 & 0,834 & 0,903 & 0,935 & 0,766 & 0,913 \\
\hline $\begin{array}{l}\text { 18. En general estoy satisfecho con } \\
\text { mi vida en este momento (P/SV) }\end{array}$ & 0,769 & 0,919 & 0,725 & 0,809 & 0,902 & 0,935 & 0,771 & 0,913 \\
\hline $\begin{array}{l}\text { 19. Creo que tengo un propósito en } \\
\text { el mundo (P/SV) }\end{array}$ & 0,706 & 0,920 & 0,642 & 0,769 & 0,904 & 0,936 & 0,718 & 0,913 \\
\hline $\begin{array}{l}\text { 20. A mis años, mi vida es mejor de } \\
\text { lo que esperaba (G) }\end{array}$ & 0,598 & 0,922 & 0,551 & 0,648 & 0,906 & 0,938 & 0,590 & 0,916 \\
\hline
\end{tabular}


quicos", "gerotrascendencia”, "espiritualidad" y "propósito/satisfacción vital". Igualmente, el modelo hipotetiza que las dimensiones "gerotrascendencia" y "propósito/satisfacción vital” convergen en la dimensión gerovida. Los resultados obtenidos se muestran en la Figura 1 , donde se muestran los resultados de análisis factorial. Dichos resultados confirman la ade- cuación de un modelo de envejecimiento exitoso basado en las cinco dimensiones originales del inventario se ajusta a los datos. Los valores de las cargas factoriales tienen un mínimo de 0,55 y un máximo de 0,98 . Los indicadores de ajuste arrojaron los siguientes valores: $\chi^{2}=575,647$; $\mathrm{p}<0,01$; RMSEA $=0,059$; $\mathrm{CFI}=0,956$; AGFI $=$ 0,904; NNFI $=0,95 ; \mathrm{IFI}=0,956$ (Figura 1$)$.

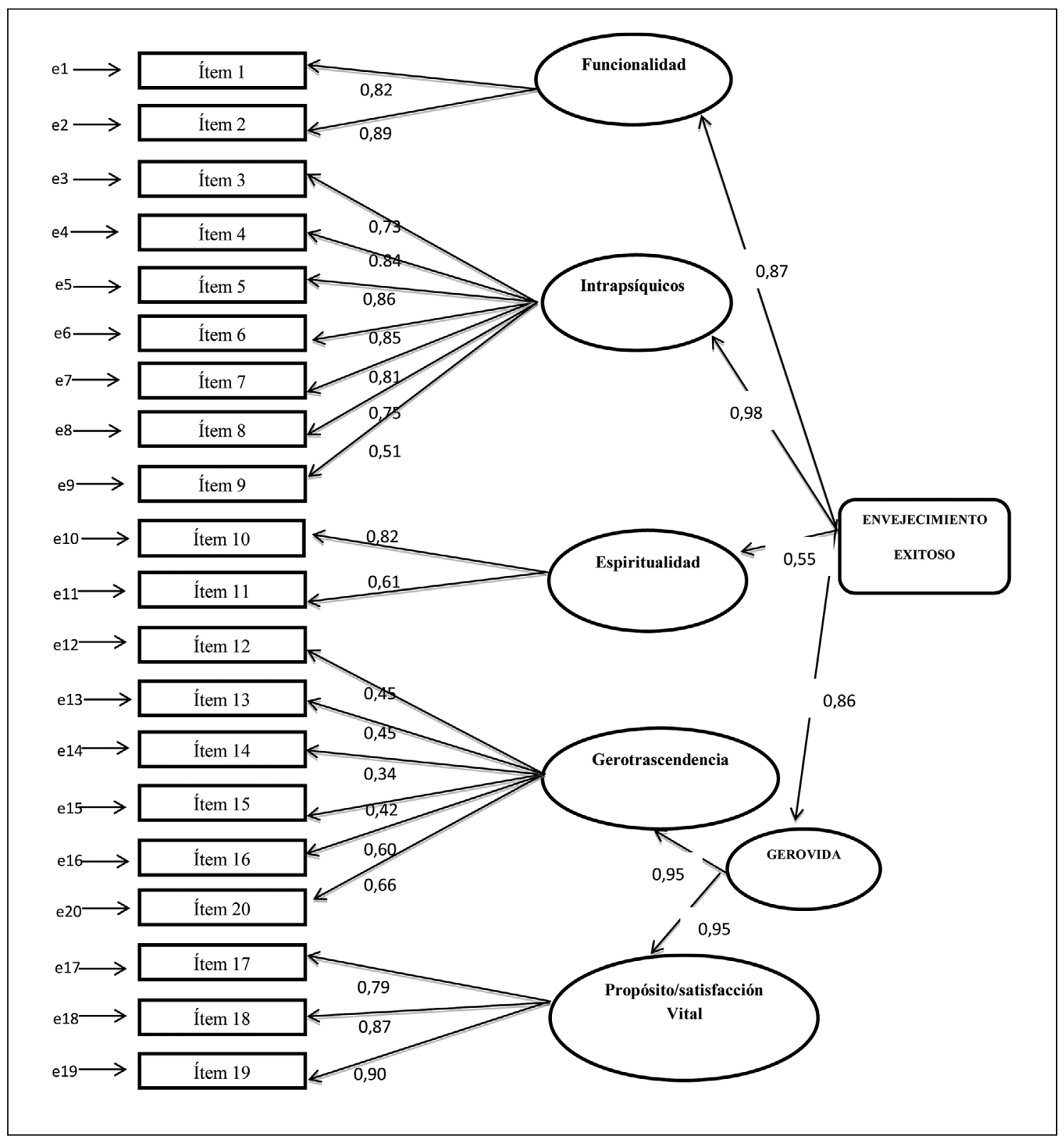

Figura 1. Modelo conceptual de las relaciones entre los ítems y dimensiones del SAI. 


\section{Discusión}

El objetivo principal de este estudio fue evaluar las propiedades psicométricas del Inventario de Envejecimiento Exitoso (SAI) en adultos mayores chilenos. Los resultados obtenidos en el análisis factorial realizado confirman el modelo original basado en cinco dimensiones o factores ("mecanismos de desempeño funcional", factores intrapsíquicos", "gerotrascendencia", "espiritualidad" y "propósito/satisfacción vital" $)^{13}$. De igual manera, los indicadores de consistencia interna obtenidos para el SAI en nuestra muestra fueron satisfactorios y son similares a los obtenidos en investigaciones previas ${ }^{16,17}$. En síntesis la fiabilidad de instrumento tanto para la escala general como en sus dimensiones es satisfactoria. Estos resultados permiten utilizar con confianza el cuestionario en población chilena, considerando la notable heterogeneidad de este grupo de edad. Teniendo en cuenta que los resultados no difieren en significativamente en función del sexo y de la pertenencia étnica. Igualmente, en este estudio se cuenta con una muestra que refleja la diversidad étnica propia de la población de personas mayores en Chile, incorporando específicamente personas pertenecientes al pueblo originario Aymara que residen en el extremo norte de país. Conviene destacar que se trata del primer cuestionario de envejecimiento exitoso validado en Chile, lo que facilitará que la investigación futura en nuestro país ahonde en esta línea fundamental en la investigación internacional. En este punto nuestros resultados sugieren la posibilidad de generar en Chile datos comparables con los obtenidos en otros países y contextos culturales.

En este contexto general, es preciso tomar en consideración las limitaciones del presente estudio. En primer lugar, son necesarias investigaciones que desarrollen y completen el proceso de validación del instrumento. En su formato original (en inglés) ${ }^{13}$, el SAI fue validado utilizando los procedimientos al uso, asegurando los diferentes ámbitos de fiabilidad y validez del inventario (validez aparente, de constructo y convergente). El presente artículo ofrece una adaptación del SAI al entorno cultural chileno. Así, los resultados del análisis factorial confirmatorio realizado muestran que las propiedades psicométricas son satisfactorias. Ahora bien, ulteriores investigaciones deben profundizar en diversos aspectos de la validación de la versión en español del inventario, realizando análisis detallados de la validez del mismo en sus diversas dimensiones. En segundo lugar, y de manera relacionada, son necesarios estudios que analicen la validez transcultural del SAI en el marco de la diversidad étnica de personas mayores chilenas. Más aún si se tiene en consideración que algunas preguntas del SAI pudiesen resultar sensibles a la diversidad cultural chilena. En efecto, el presente trabajo pone de manifiesto la necesidad de realizar adaptaciones dirigidas a la aplicación del instrumento entre personas mayores de las diferentes etnias originarias de nuestro país, y de manera específica para las personas pertenecientes al pueblo Aymara. En tercer lugar, hay que tener en cuenta que en nuestro estudio el inventario fue aplicado a través de entrevista personal. Esta circunstancia invita a tener en cuenta los diversos sesgos del entrevistador (efecto Rosenthal). Por ejemplo, el conocimiento de los objetivos del estudio por parte de los/as entrevistadores puede llevar a que, intencionalmente o no, puedan ofrecerse señales, matices de voz, expresiones faciales o corporales, proporcionar indicios, etc., que potencialmente puedan influir en las respuestas. No obstante, y a pesar de este posible sesgo, las características de la muestra, compuesta por personas mayores que pueden presentar diversos problemas para la lectura, escritura y manejo del cuestionario, llevaron a estimar que esta el formato de administración a través de entrevista es el más adecuado para la población objeto de estudio.

En conclusión, el SAI es un instrumento fiable y adecuado para ser aplicado en personas mayores chilenas. De esta manera, la evaluación del envejecimiento con éxito a través de este inventario se presenta como una alternativa eficaz y rigurosa para obtener datos comparables con los procedentes de investigaciones llevadas a cabo en otras sociedades y contextos culturales. Además, este instrumento es útil para la evaluación gerontológica que tenga el objetivo de promocionar o, en su caso, preparar a las personas mayores hacia un envejecimiento exitoso, ya sea mejorando la dieta, el ejercicio físico, los hábitos personales o a través de la promoción de recursos psicosociales de adaptación al proceso de envejecimiento. 


\section{Referencias}

1. Rowe JW, Kahn RL. Human aging: usual and successful. Science 1987; 237: 143-9.

2. Rowe JW, Kahn RL. Successful aging. Gerontologist 1997; 37 (4): 433-40.

3. Flood M. Successful aging: a concept analysis. J Theory Constr Test 2002; 6 (2): 105-8.

4. Cosco TD, Prina AM, Perales J, Stephan BC, Brayne C. Operational definitions of successful aging: a systematic review. Int Psychogeriatri 2014; 26 (3): 373-81.

5. Cheng ST. Defining successful aging: the need to distinguish pathways from outcomes. Int Psychogeriatr 2014; 26 (4): 527-31.

6. Kanning M, Schlicht W. A bio-psycho-social model of successful aging as shown through the variable "physical activity”. Eur Rev Aging Phys Act 2008; 5(2): 79-87.

7. Fernández-Ballesteros R, Zamarrón MD, López MD, Molina MA, Díez J, Montero P , et al. Envejecimiento con éxito: criterios y predictores. Psicothema 2010; 22 (4): 641-7.

8. Carrascosa-Gil R, Vázquez-Calatayud M, Canga-Armayor AD. Successful aging: un enfoque holístico. Gerokomos 2010; 21 (4): 146-52.

9. Li CT, Lin CH, Lin WY, Liu CS, Chang CK, Meng NH, et al. Successful aging defined by health-related quality of life and its determinants in community-dwelling elders. BMC Public Health 2014; 14 (1): 1-8.

10. Hilton JM, González CA, Saleh M, Maitoza R, Anngela-cole L. Perceptions of successful aging among older latinos, in cross-cultural context. J Cross Cult Gerontol 2012; 27(3): 183-99.

11. Liffiton JA, Horton S, Baker J, Weir, PL. Successful aging: how does physical activity influence engagement with life? Eur Rev Aging Phys Act 2012; 9(2): 103-8.

12. Depp CA, Jeste DV. Definitions and predictors of successful aging: a comprehensive review of larger quantitative studies. Am J Geriatr Psychiatry 2006; 14 (1): 6-20.

13. Troutman M, Nies MA, Small S, Bates A. The development and testing of an instrument to measure successful aging. Res Gerontol Nurs 2011; 4 (3): 221-32.

14. Crowther MR, Parker MW, Achenbaum WA, Larimore WL, Koenig HG. Rowe and Kahn's model of successful aging revisited: positive spirituality-the forgotten factor.
Gerontologist 2002; 42 (5): 613-20.

15. McCann P, Ward L, Winefield H. Successful ageing by whose definition? Views of older, spiritually affiliated women. Australas J Ageing 2008; 27 (4): 200-4.

16. Troutman M, Nies MA, Bentley M. Measuring successful aging in southern black older adults. Educ Gerontol 2010; 37 (1): 38-50.

17. Kozar-Westman M, Troutman-Jordan M, Nies MA. Successful aging among assisted living community older adults. J Nurs Scholarsh 2013; 45 (3): 238-46.

18. Howie LO, Troutman-Jordan M, Newman AM. Social support and successful aging in assisted living residents. Educ Gerontol 2014; 40 (1): 61-70.

19. Cho J, Martin P, Poon LW, Georgia Centenarian Study. Successful aging and subjective well-being among oldest-old adults. Gerontologist 2015; 55 (1): 132-43.

20. Jopp D, Rott C. Adaptation in very old age: exploring the role of resources, beliefs, and attitudes for centenarians' happiness. Psychol Aging 2006; 21(2): 266-80.

21. Arechabala MC, Miranda C. Validación de una escala de apoyo social percibido en un grupo de adultos mayores adscritos a un programa de hipertensión de la región Metropolitana. Cienc enferm 2002; 8(1): 49-55.

22. Espinoza I, Osorio P, Torrejón MJ, Lucas-Carrasco R, Bunout D. Validación del cuestionario de calidad de vida (WHOQOL-BREF) en adultos mayores chilenos. Rev Med Chile 2011; 139 (5): 579-86.

23. Herrera MS, Saldías P, Testa N. Validación de un test breve para el diagnóstico de capacidad funcional en adultos mayores en Chile. Rev Med Chile 2014; 142 (9): 1128-35.

24. Steiger JH. Understanding the limitations of global fit assessment in structural equation modeling. Pers Individ Dif 2007; 42(2): 893-8.

25. Hu LT, Bentler PM. Cutoff criteria for fit indexes in covariance structure analysis: Conventional criteria versus new alternatives. Structural Equation Modeling 1999; 6 (1): 1-55.

26. Kautín MA. Estímulos focales y contextuales en respuestas adaptativas para el envejecimiento exitoso en adultos mayores. Tesis doctoral. Universidad Autónoma de Nueva León. Programa de doctorado: Ciencias de la Enfermería. (Consultado el 9 de noviembre de 2016). Disponible en http://eprints.uanl.mx/2998/1/1080227492. pdf 